\title{
Synthesis, Characterization and Photoluminescence of Terbium (III) Complex with the Schiff Base
}

\author{
YU Xiang \\ College of Chemical and \\ Environmental Engineering, \\ Guangdong University of \\ Petrochemical Technology, \\ Guangdong Maoming, China \\ yygdupt_mmc@126.com
}

\author{
YU Zhidong \\ College of Chemical and \\ Environmental Engineering, \\ Guangdong University of \\ Petrochemical Technology, \\ Guangdong Maoming, China
}

\author{
ZHOU Rujin \\ College of Chemical and \\ Environmental Engineering, \\ Guangdong University of \\ Petrochemical Technology, \\ Guangdong Maoming, China
}

\begin{abstract}
A new terbium (III) complex of the Schiff base (HL) derived from1-phenyl-3-furyl-4-benzoyl-4, 5-dihydropyrazol-5one (PFBP) and 2-aminobenzimidazole (2-AB) has been synthesized and investigated by photolum-inescence spectroscopy. The molecular structure was characterized by IR spectra, UV spectra and 1H NMR spectra; moreover, a high performance liquid chromatography-mass spectrometry (HPLC-MS) method was developed for the analysis of synthetic system. The integrated analytical results show that the new ligand coexists in keto-form structure and enol-form structure under the measuring conditions, the keto-form structure may change into the enolform structure during the coordination process. The photoluminescence spectra show that the complex exhibits the strong green fluorescence.
\end{abstract}

Keywords-Schiff base; terbium (III) complex; HPLC-MS; photoluminescence

\section{INTRODUCTION}

The organic rare earth complexes have intensively been studied owing to their good luminescence properties[1-3], bioinorganic sensors[4] and organic electroluminescent devices (OELD)[5-7]. Because of the shielding effect of $5 \mathrm{~s}$ and $5 \mathrm{p}$ electrons, $4 \mathrm{f}$ electrons of the rare earth ions do not participate bonding with the ligands directly, the $4 \mathrm{f} \rightarrow 4 \mathrm{f}$ transitions act as those of free atoms, resulting in very narrow absorption and emission bands. This leads to good luminescent. The complexes of $\mathrm{Sm}(\mathrm{III}), \mathrm{Eu}(\mathrm{III})$ and $\mathrm{Tb}(\mathrm{III})$ with $\beta$-diketones or other ligands which can absorb and transfer the light energy to the central metals give very strong luminescent intensity due to the increase of absorbability[8].

4-Acyl pyrazolones are good extractants of rare earth ions. In addition, 4-acyl pyrazolones can form a variety of Schiff bases which have been reported to be superior ligands of rare earth ions ${ }^{[9,10]}$. In the hunt for obtaining luminescence and amplifying the scope of 4-acyl pyrazolone derivatives coordinated with rare earth, the synthesis of new rare earth complex of 4-acyl pyrazolone derivatives, terbium (III) complex with the Schiff base derived from 1-phenyl-3-furyl-4benzoyl-4, 5-dihydropyrazol-5-one and 2-aminobenzimidazole was performed. Their coordination structure and luminescent

This work was supported by Guangdong Provincial Natural Science Foundation Project, China (9152500002000015). property w ere studied.

\section{EXPERIMENTAL}

\section{A. Materials and methods}

The compound 1-phenyl-3-furyl-5-pyrazolone (PFP) was synthesized according to the literature [11]. The 2-AB was synthesized according to the literature [12].

Terbium was estimated by the titrimetric method using EDTA. LC-MS analysis was performed on a model Agilent 1100 LC-MSD-TRAP. Infrared (IR) spectra were recorded on a Avatar 370 FT-IR spectrometer as $\mathrm{KBr}$ pellets. 1H NMR spectra were recorded on a model AV 300 Bruker nuclear magnetic resonance instrument. Electronic spectra were recorded on a model U-3010 Hitachi spectrophotometer. Photoluminescence were made on a Hitachi F-4500 spectrometer equipped with quartz curettes of $1 \mathrm{~cm}$ path length.

\section{B. Synthesis of PFBP}

PFP (2.26 g, 0.01 mol) was dissolved in hot dioxane $(10 \mathrm{~mL})$ in a flask equipped with a stirrer, separating funnel and reflux condenser. Calcium hydroxide $(1.48 \mathrm{~g}, 0.02 \mathrm{~mol})$ was added to this solution, followed by benzoyl chloride $(1.40 \mathrm{~g}, 0.01 \mathrm{~mol})$ added dropwise with precaution. After the complete addition, the reaction mixture was refluxed for half an hour and then it was poured into dilute hydrochloric acid $(10 \mathrm{~mL}, 2 \mathrm{M})$. The flaxen needle shaped crystals (PFBP) thus obtained were separated by filtration and recrystallized from an acidified methanol-water mixture.

\section{Synthesis of the Schiff base (HL)}

Anhydrous alcohol (10mL) was added to a $150 \mathrm{~mL}$ Erlenmeyer flask containing PFBP $(3.30 \mathrm{~g}, 0.01 \mathrm{~mol})$ equipped with a stirrer, separating funnel and reflux condenser. After heating up the flask to dissolve the PFBP completely, ethyl alcohol solution containing $2-\mathrm{AB}(1.33 \mathrm{~g}, 0.01 \mathrm{~mol})$ was added dropwise with precaution. Then the reaction mixture was refluxed for four hours. Yellow crystal matter was obtained after filtrating, washing by anhydrous alcohol, recrystallizing two times in acetone and drying. 


\section{Synthesis of terbium complex $\left[\mathrm{TbL}_{2} \cdot 2 \mathrm{H}_{2} \mathrm{O}\right]$}

The HL (0.445 g, 1mmol) dissolved in hot anhydrous alcohol $(8 \mathrm{~mL})$ was added dropwise into an anhydrous alcohol of terbium nitrate $(0.227 \mathrm{~g}, 0.50 \mathrm{mmol})$ with stirring. After the complete addition, $\mathrm{NaOH}$ solution $(\mathrm{v}+\mathrm{v}=1+4)$ was added to adjust the $\mathrm{pH}=5 \sim 6$ and the reaction mixture was refluxed for $6 \mathrm{~h}$. The bisque solid matter was obtained after filtrating, washing, recrystallizing two times in acetone and drying in air. Synthetic route was presented in Fig. 1.

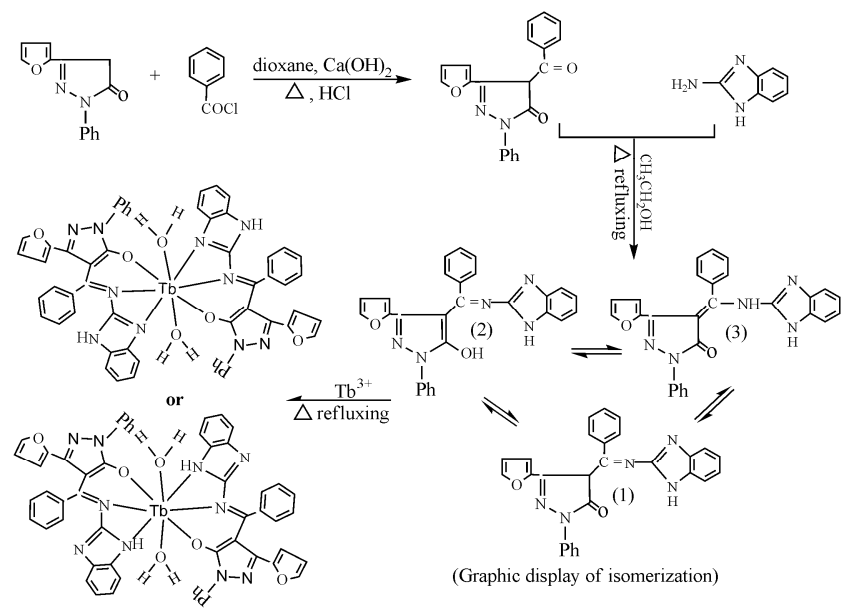

Fig. 1 Synthetic route of terbium complex.

\section{E. LC-MS analysis for the reaction system of terbium complex $\left[\mathrm{TbL}_{2} \cdot 2 \mathrm{H}_{2} \mathrm{O}\right]$}

The experiment was carried out with a LC-MSD-Trap system being comprised of a quaternary pump, vacuum degasser, thermostated column compartment. After the base line became stable, samples were injected according to the sequence of initial-term, medium-term and final-term reaction solution. Ion peaks of MS and their intensity data corresponding to the retention time of chromatograms are listed as: t0.78: $(+)[\mathrm{M}+\mathrm{H}]+=446.2(0.8 \times 106),[\mathrm{M}+\mathrm{Na}]+=468.0$ $(0.3 \times 106),[\mathrm{M}-\mathrm{H}+2 \mathrm{Na}]+=490.2(0.1 \times 106),[\mathrm{M}+\mathrm{K}]+=484.4$ $(0.15 \times 106),[\mathrm{M}-\mathrm{H}+\mathrm{Na}+\mathrm{K}]+=506.1(0.1 \times 106),(-)[\mathrm{M}-\mathrm{H}]+=$ $444.0(0.9 \times 107) ; \quad \mathrm{t} 6.50:(+)[\mathrm{M}+\mathrm{H}]+=446.0 \quad(0.3 \times 106)$, $[\mathrm{M}+\mathrm{Na}]+=468.1(0.6 \times 106),[\mathrm{M}-\mathrm{H}+2 \mathrm{Na}]+=490.1(0.2 \times 106)$, $[\mathrm{M}+\mathrm{K}]+=484.2(0.1 \times 106),(-)[\mathrm{M}-\mathrm{H}]+=444.2(0.07 \times 104) ;$ t7.19: $(+)[\mathrm{M}+\mathrm{H}]+=446.0(0.2 \times 106),[\mathrm{M}+\mathrm{Na}]+=468.1$ $(0.5 \times 106),[\mathrm{M}+\mathrm{K}]+=484.1(0.15 \times 105),(-)[\mathrm{M}-\mathrm{H}]+=444.0$ $(0.1 \times 104)$; t11.42: $(+)[\mathrm{M}-2 \mathrm{H} 2 \mathrm{O}+\mathrm{H}]+=1048.6(0.5 \times 105)$,
$[\mathrm{M}-2 \mathrm{H} 2 \mathrm{O}+\mathrm{Na}]+=1070.4(0.3 \times 105),[\mathrm{M}-2 \mathrm{H} 2 \mathrm{O}+\mathrm{K}]+=1086.5$ $(0.1 \times 105)$.

\section{RESULTS AND DISCUSSION}

\section{A. Electronic spectra}

The electronic spectra of HL and its terbium complex were recorded in DMF solution of $1 \times 10^{-5} \mathrm{~mol} \cdot \mathrm{L}^{-1}$ and the data are presented in Table 1. The four absorption peaks of HL at ultraviolet region at 209, 222, 236 and $330 \mathrm{~nm}$ are assigned to the band of $\pi-\pi^{*}$ or $n-\pi^{*}$ transitions of aromatic ring, furan ring, benzimidazole ring and two imido groups. Compared with HL, the first peak of terbium complex unchanges basically and the second, the third and the fourth peak exhibit the red shifts in various degrees. These are possibly concerned with the coordination between terbium central ion and two $\mathrm{N}$ atoms of imido group and benzimidazole ring of HL, which causes the polarization of $\mathrm{C}=\mathrm{N}$ bond and effects energy levels of the related conjugated molecular orbitals.

\section{B. IR spectra}

The characteristic absorption bands data of IR spectra of $\mathrm{HL}$ and its terbium complex recorded in the range of 4000-400 $\mathrm{cm}^{-1}$ are shown in Table 1 . The broad $v_{\mathrm{O}-\mathrm{H} . . . \mathrm{N}}$ band formed by enolic $\mathrm{OH}$ of pyrazolone ring and $\mathrm{N}$ atom of imido group was observed at $3528 \mathrm{~cm}^{-1}$ for $\mathrm{HL}$, which shows that the HL coexists in keto-form structure and enol-form structure. This inferred conclusion is in accord with the one enduced from LCMS and ${ }^{1} \mathrm{H}$ NMR analysis. Compared with $\mathrm{HL}, \mathrm{H}_{2} \mathrm{O}$ band appears at $3618 \mathrm{~cm}^{-1}$ after formation of terbium complex, which indicates that $\mathrm{O}$ atom of $\mathrm{H}_{2} \mathrm{O}$ participates in the coordination process. That the absorption band of $v_{\mathrm{C}=\mathrm{O}}$ shifts from $1630 \mathrm{~cm}^{-1}$ of HL to $1619 \mathrm{~cm}^{-1}$ of terbium complex shows that the keto-form structure may change into the enol-form for $\mathrm{HL}$ during the coordination process, moreover the vibrational bands of $v_{\mathrm{Tb}-\mathrm{O}}$ appears at $506 \mathrm{~cm}^{-1}$, which shows that $\mathrm{O}$ atom of hydroxyl group of enol-form structure participates in the coordination; That the absorption band of $v_{\mathrm{C}=\mathrm{N}}$ shifts from 1563 $\mathrm{cm}^{-1}, 1552 \mathrm{~cm}^{-1}$ and $1546 \mathrm{~cm}^{-1}$ of HL to $1560 \mathrm{~cm}^{-1}, 1548 \mathrm{~cm}^{-1}$ and $1539 \mathrm{~cm}^{-1}$ of terbium complex and the vibrational band of $v_{\mathrm{Tb}-\mathrm{N}}$ appears at $492 \mathrm{~cm}^{-1}$ show that two $\mathrm{N}$ atoms of imido group and benzimidazole ring participate in the coordination ${ }^{[13]}$. The analytical results show that the keto-form structure of HL may change into the enol-form structure during the coordination and the ligand bonds with terbium central ion by two $\mathrm{O}$ atoms of hydroxyl group of pyrazolone ring and $\mathrm{H}_{2} \mathrm{O}$ and two $\mathrm{N}$ atoms of imido group and benzimadazole ring.

TABLE 1 UV SPECTRAL AND IR DATA OF THE LIGAND AND ITS TERBIUM COMPLEX

\begin{tabular}{|c|c|c|c|c|c|c|c|c|c|c|}
\hline Complex & & & & & UV[nm] & & $\operatorname{IR}\left[\mathrm{cm}^{-1}\right.$ & & & \\
\hline & $\lambda_{1}$ & $\lambda_{2}$ & $\lambda_{3}$ & $\lambda_{4}$ & $v_{O-H \ldots N}$ & $v_{C=O}\left(v_{C=C}\right)$ & $v_{C=N}$ & $v_{C-O}$ & $v_{T b-O}$ & $v_{T b-N}$ \\
\hline $\mathrm{HL}$ & 209 & 222 & 236 & 330 & 3128 & 1630 & 156315521546 & 1480 & & \\
\hline $\mathrm{TbL}_{2} \cdot 2 \mathrm{H}_{2} \mathrm{O}$ & 209 & 224 & 244 & 342 & 3618 & 1619 & 156015481539 & & 506 & 492 \\
\hline
\end{tabular}

\section{C. ${ }^{1} H N M R$ of $H$}

The ${ }^{1} \mathrm{H}$ NMR spectra of $\mathrm{HL}$ was recorded in $\mathrm{CDCl}_{3}$ at room temperature regarding TMS as internal standard. From
Fig.2, The multiplets of 17 protons appearing at the range of $\delta$ 7.112-8.385 are assigned to 17 protons of benzene ring, furan ring and benzimidazole ring. The singlet whose integral area is 
good equivalent to one proton appearing at $\delta 12.852$ corresponds to the proton of $-\mathrm{NH}$ of benzimidazole ring. Another weak and broad singlet is observed at $\delta 13.333$ at low field region. It can be assigned to either to $\mathrm{OH}$ or $\mathrm{NH}$, in either case it is strongly deshielded because of hydrogen bonding with the other atom $(\mathrm{N} / \mathrm{O})^{[14]}$. The sum of its integral area of 0.8800 and the integral area of 0.1150 of weaker singlet at $\delta$ 6.784 perfectly matches with one proton, so the proton appeared at $\delta 6.784$ is assigned to the proton of methylene of pyrazolone ring, which suggests that HL coexists in keto-form structure and in enol-form structure under the experimental conditions. This conclusion is in accord with the one enduced from IR spectra and LC-MS analysis.
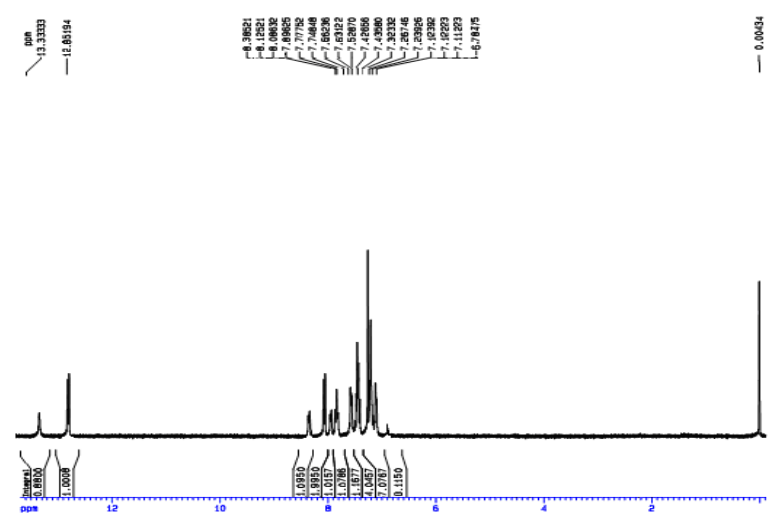

Fig. $2{ }^{1} \mathrm{H}$ NMR of $\mathrm{HL}$

\section{Analysis of LC-MS testing for synthetical system of terbium complex}

From Fig. 2, three absorption peaks appeared at $t_{0.78}, t_{6.50}$ and $t_{7.19}$ in initial-term reaction system respectively. Under positive ion scanning mode, $[\mathrm{M}+\mathrm{H}]^{+}$of $\mathrm{m} / \mathrm{z} 446$ is base peak among ion peaks of MS corresponding with $\mathrm{t}_{0.78}$, but $[\mathrm{M}+\mathrm{Na}]^{+}$ of $\mathrm{m} / \mathrm{z} 468$ is base peak among ion peaks of MS corresponding with $t_{6.50}$ and $t_{7.19}$; Under negative ion scanning mode, very strong $[\mathrm{M}-\mathrm{H}]^{-}$ion peak of $\mathrm{m} / \mathrm{z} 444$ appeared under injection volume of $2 \mu \mathrm{L}$ at $\mathrm{t}_{0.78}$, but weak signal of this ion peak was observed under injection volume of $20 \mu \mathrm{L}$ at $\mathrm{t}_{6.50}$ and $\mathrm{t}_{7.19}$, which results from that the hydroxyl of enol-form structure is easier to lose one proton to form negative ion. The absorption peak corresponding to $\mathrm{t}_{0.78}$ is inferred to be the one corresponding to enol-form structure, but two absorption peaks corresponding to $t_{6.50}$ and $t_{7.19}$ are two ones corresponding to two keto-form structures. They may be inferred to be two absorption peaks corresponding to keto-form structure (3) and (1) orderly from the polarity of molecular structure. According to mass spectra data corresponding to the absorption peaks of medium-term reaction system, mass spectra ion peaks corresponding to HL and target product appeared, of which mass spectra ion peaks corresponding to enol-form structure and two keto-form structures of HL were all observed. Three ion peaks are confirmed to be $\left[\mathrm{TbL}_{2} \cdot 2 \mathrm{H}_{2} \mathrm{O}-2 \mathrm{H}_{2} \mathrm{O}+\mathrm{H}\right]^{+}$, $\left[\mathrm{TbL}_{2} \cdot 2 \mathrm{H}_{2} \mathrm{O}-2 \mathrm{H}_{2} \mathrm{O}+\mathrm{Na}\right]^{+}$and $\left[\mathrm{TbL}_{2} \cdot 2 \mathrm{H}_{2} \mathrm{O}-2 \mathrm{H}_{2} \mathrm{O}+\mathrm{K}\right]^{+}$ respectively. The varying of absorption peak shape and the data of corresponding mass spectra show that the keto-form structure of HL may change into the enol-form structure during the formation of terbium complex and $\mathrm{O}$ atom of hydroxyl group of pyrazolone ring and $\mathrm{N}$ atom (or $\mathrm{N}$ atom of $-\mathrm{NH}$ ) of benzimidazole ring participate in the coordination by losing the proton.

\section{E. Photoluminescence studies}

The excitation spectra and the emission spectra of the complex in solid and in DMF solution are shown in Fig.3. From Fig. 3 it could be seen that the excitation wavelengths of the complex are different between in solid $(\lambda \mathrm{ex}=326 \mathrm{~nm})$ and in DMF solution $(\lambda \mathrm{ex}=380 \mathrm{~nm})$. Three characteristic bands of the central $\mathrm{Tb}^{3+}$ ion at around $494 \mathrm{~nm}, 545 \mathrm{~nm}$ and $590 \mathrm{~nm}$ were observed and assigned to the $\mathrm{Tb}^{3+} \mathrm{f}-\mathrm{f}$ transition of ${ }^{5} \mathrm{D}_{4} \rightarrow{ }^{7} \mathrm{~F}_{6},{ }^{5} \mathrm{D}_{4} \rightarrow{ }^{7} \mathrm{~F}_{5}$ and ${ }^{5} \mathrm{D}_{4} \rightarrow{ }^{7} \mathrm{~F}_{4}$ respectively. It were showed the relative intensity of the complex at $545 \mathrm{~nm}$ is strong either in solid or in DMF solution, this is related to the efficiency of the intramolecular energy transfer between the triplet level of ligand and the emitting level of the ion, which depends on the energy gap between the two levels. Probably the energy gap between the ligand triplet levels and the emitting level of terbium favor to the energy transfer process for terbium.

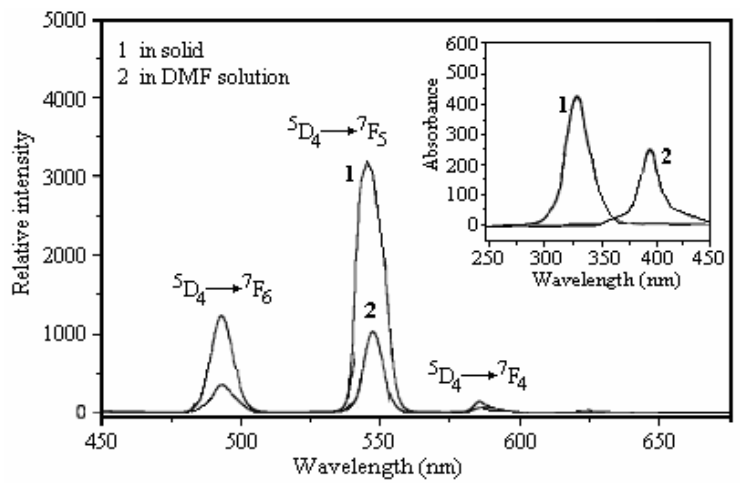

Fig. 3 Photoluminescence spectraof the complex

\section{CONCLUSION}

A new 4-acyl pyrazolone-based Schiff base ligand and its terbium (III) complex was synthesized in this paper. The results of LC-MS analysis integrating with other characterization means else show that the new ligand coexists in keto-form structure and enol-form structure under the measuring condition, the keto-form structure may change into the enol-form structure during the coordination and the ligand bonds with terbium central ion by two $\mathrm{O}$ atom of hydroxyl group of pyrazolone ring and $\mathrm{H}_{2} \mathrm{O}$ and $\mathrm{N}$ atom of imine group and $\mathrm{N}$ atom (or $\mathrm{N}$ atom of $-\mathrm{NH}$ ) of benzimidazole ring by losing the proton. The photoluminescence data show that the complex exhibits three characteristic emission peaks of $\mathrm{Tb}^{3+}$ ion both in solid and in DMF solution. That the peak's relative intensity at $545 \mathrm{~nm}$ is strong suggests that the new ligand can activate efficiently the luminescence of the $\mathrm{Tb}^{3+}$ ion, making the complex a potential candidate as emitter in photonic systems. 


\section{REFERENCES}

[1] A. Listkowski, M. Pietraszkiewicz, G. Accorsi, J. Mohanraj, "Solid state photoluminescence of novel lanthanide complexes based on 4benzoylpyrazolone Schiff base," Synth. Met. Vol. 160, pp. 2377-2380, November 2010

[2] C.R. Bhattacharjee, G. Das, P. Goswami, P. Mondal, S.K. Prasad, D.S.S. Rao, " Novel photoluminescent lanthanidomesogens forming bilayer smectic phase derived from blue light emitting liquid crystalline, one ring O-donor Schiff-base ligands," Polyhedron, Vol. 30, pp.1040-1047, April 2011.

[3] A. Kagkelar, V. Bekiari, E. Stathatos, G.S. Papaefstathiou, C.P. Raptopoulou, T.F. Zafiropoulos, et al, "Photoluminescence and electroluminescence by gallium(III) complexes of N-salicylidene-oaminophenol and its derivatives," J Lumin., Vol. 129, pp. 578-583, May 2009.

[4] M. Altaf, H. Stoeckli-Evans, "Nickel(II) and copper(II) complexes of 2(2-pyridyl)benzimidazole: synthesis and structural characterization," Transition Met. Chem. Vol. 34, pp.613-620, June 2009.

[5] W.G. Quirino, C. Legnani, M. Cremona, P.P. Lima, S.A. Junior, O.L. Malta, "White OLED using $\beta$-diketones rare earth binuclear complex as emitting layer," Thin Solid Films, Vol. 494, pp.23-27, January 2006.

[6] R. Reyes, M. Cremona, E.E.S. Teotonio, H.F. Brito, O.L. Malta, "Voltage color tunable OLED with $(\mathrm{Sm}, \mathrm{Eu})-\beta$-diketonate complex blend," Chem. Phys. Lett. Vol. 396, pp.54-58, September 2004.

[7] F.F. Chen, Z.Q. Bian, C.H. Huang, "Progresses in electroluminescence based on europium(III) complexes," J. Rare Earth. Vol. 27, pp.345-355, June 2009 .
[8] H.G. Liu, S. Park, K. Jang, W.S. Zhang, H.J. Seo, Y.I. Lee, "Different photoluminescent properties of binary and ternary europium chelates doped in PMMA," Mater. Chem. Phys. Vol. 82, pp. 84-92, September 2003.

[9] Y.L. Zhao, D.J. Zhou, G.Q. Yao, C.H. Huang, "Strong Luminescent Terbium(III)-Pyrazolone Complexes and Their Langmuir-Blodgett Films," Langmuir Vol. 13, pp. 4060-4065, July 1997.

[10] Z.G. Yu, B. Liu, Z.H. Jiang, "Characterization by Spectra and Identification by HPLC-MS of Schiff Base Derived from 1-Phenyl-3methyl-4-naphthylaceto-4,5-dihydropyrazol-5-one (PMNAP) and 2Aminobenzimidazole (2-AB) and Its Transitional Metal Complexes," Chinese J. Org. Chem. Vol. 29, pp. 1217-1222, August 2009.

[11] J.Z. Li, H.Q. Zhang, H.X. Li, P.Z. Che, T.C. Wang, "1-(4Chlorophenyl)-4-(2-furoyl)-3-(2-furyl)-1H-pyrazol-5-ol," Acta Cryst E, Vol. 63, pp.1289-1290, March 2007.

[12] W.Z. Lv, B Liu, S.H. Wei, Y. Gao, 'Synthesis and characteristics of 2aminobenzimidazole under microwave irradiation," Chem. Reagents Vol. 28, pp. 751-752, December 2006.

[13] S.P. Mital, R.V. Singh, J.P. Tandon, "Imine derivatives of lanthanons," Curr. Sci. Vol. 49,pp.130-132, April 1980.

[14] R.N. Jadeja, J.R. Shah, E. Suresh, P. Paul, "Synthesis and structural characterization of some Schiff bases derived from 4[\{(aryl)imino\} ethyl]-3-methyl-1-(4'-methylphenyl)-2-pyrazolin-5-one and spectroscopic studies of their $\mathrm{Cu}(\mathrm{II})$ complexes," Polyhedron Vol. 23, pp. 2465-2474, October 2004. 